\title{
Josephson Effect due to Odd-Frequency Pairs in Diffusive Half Metals
}

\author{
Yasuhiro Asano, ${ }^{1}$ Yukio Tanaka, ${ }^{2}$ and Alexander A. Golubov ${ }^{3}$ \\ ${ }^{1}$ Department of Applied Physics, Hokkaido University, Sapporo 060-8628, Japan \\ ${ }^{2}$ CREST-JST and Department of Applied Physics, Nagoya University, Nagoya 464-8603, Japan \\ ${ }^{3}$ Faculty of Science and Technology, University of Twente, 7500 AE, Enschede, The Netherlands
}

(Received 21 September 2006; published 8 March 2007)

\begin{abstract}
Motivated by a recent experiment [Keizer et al., Nature (London) 439, 825 (2006)], we study the Josephson effect in superconductor/diffusive half metal/superconductor junctions using the recursive Green function method. The spin-flip scattering at the junction interfaces opens the Josephson channel of the odd-frequency spin-triplet Cooper pairs. As a consequence, the local density of states in a half metal has a large peak at the Fermi energy. Therefore the odd-frequency pairs can be detected experimentally by using the scanning tunneling spectroscopy.
\end{abstract}

DOI: 10.1103/PhysRevLett.98.107002

PACS numbers: 74.50.+r, 74.25.Fy, 74.70.Tx

Ferromagnetism and spin-singlet superconductivity are competing orders against each other because the exchange field breaks down the spin-singlet pairs. The Cooper pairs change their original face to survive in the exchange field. The Fulde-Ferrell-Larkin-Ovchinnikov [1,2] state and the proximity effect in ferromagnets [3-10] are typical examples. In weak exchange fields, the pairing function oscillates and changes its sign in real space. As a consequence, superconductor/ferromagnet/superconductor (SFS) junctions undergo the $0-\pi$ transition with varying length of a ferromagnet or temperature. In a sufficiently strong exchange field, however, the spin-singlet Cooper pairs would disappear.

Half metal is an extreme case of a completely spin polarized material because its electronic structure is insulating for one spin direction and metallic for the other. At first thought, the spin-singlet Cooper pairs would not be able to penetrate into half metals. However, a recent experiment [11] showed the existence of Josephson coupling in superconductor/half metal/superconductor (S/HM/S) junctions. Thus one has to seek a new state of Cooper pairs in half metals attached to spin-singlet superconductors. Prior to the experiment [11], Eschrig et al. [12] addressed this challenging issue. In the clean limit, they showed that $p$-wave spin-triplet pairs induced by spin-flip scattering at the interface can carry the Josephson current. In real S/ $\mathrm{HM} / \mathrm{S}$ junctions, however, half metals are close to the dirty limit in the diffusive transport regime; the elastic mean free path may become comparable to the coherence length and is much smaller than the size of a half metal.

In this Letter, we study the Josephson effect in SFS junctions for arbitrary magnitude of the exchange field $V_{\text {ex }}$. When $V_{\text {ex }}$ is much larger than the pair potential at zero temperature $\Delta_{0}$, the mesoscopic fluctuations of the Josephson current are much larger than its ensemble averaged value. The spin-flip scattering at the junction interfaces drastically changes the behavior of the Josephson current and the properties of Cooper pairs in a ferromagnet. In particular in diffusive S/HM/S junctions, all Cooper pairs in a half metal are in the odd-frequency spin-triplet pairing state. We propose an experimental method to detect this unusual property.

Let us consider the two-dimensional tight-binding model as shown in Fig. 1(a). The vector $\boldsymbol{r}=j \boldsymbol{x}+m \boldsymbol{y}$ points to a lattice site with $\boldsymbol{r}$ and $\boldsymbol{y}$ being unit vectors in the $x$ and $y$ directions, respectively. In the $y$ direction, we apply the periodic boundary condition for the number of lattice sites being $W$. Electronic states in superconducting junctions are described by the mean-field Hamiltonian

$$
\begin{aligned}
H_{\mathrm{BCS}}= & \frac{1}{2} \sum_{\boldsymbol{r}, \boldsymbol{r}^{\prime}}\left[\tilde{c}_{\boldsymbol{r}}^{\dagger} \hat{h}_{\boldsymbol{r}, \boldsymbol{r}^{\prime}} \tilde{c}_{\boldsymbol{r}^{\prime}}-\overline{\tilde{c}}_{\boldsymbol{r}} \hat{h}_{\boldsymbol{r}, \boldsymbol{r}^{\prime}}^{*} \overline{\tilde{c}}_{\boldsymbol{r}^{\prime}}^{\dagger}\right] \\
& +\frac{1}{2} \sum_{\boldsymbol{r} \in S}\left[\tilde{c} \dagger \hat{\Delta} \hat{\Delta} \overline{\tilde{c}}_{\boldsymbol{r}}^{\dagger}-\overline{\tilde{c}}_{\boldsymbol{r}} \hat{\Delta}^{*} \tilde{c}_{\boldsymbol{r}}\right]
\end{aligned}
$$

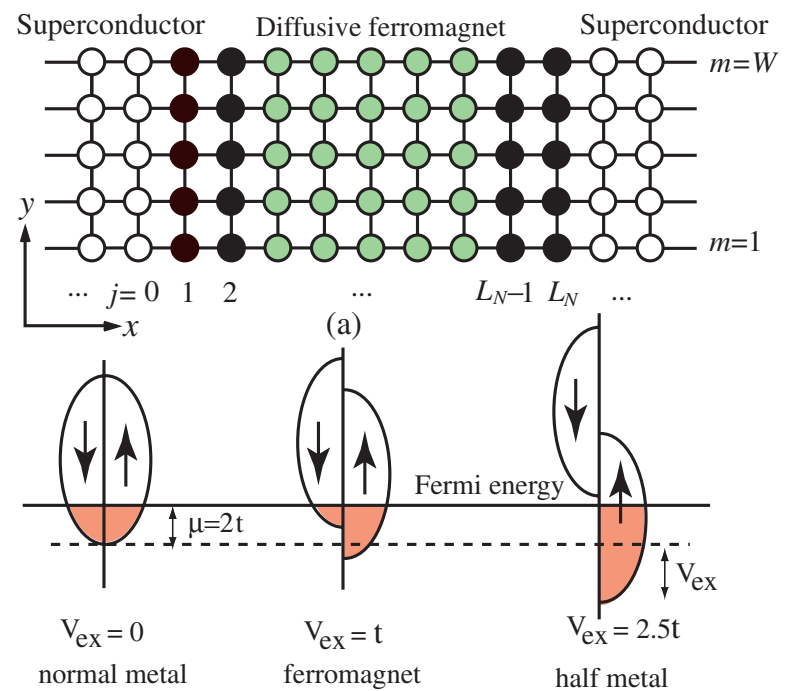

(b)

FIG. 1 (color online). (a) A schematic figure of a SFS junction on the tight-binding lattice. (b) The density of states for each spin direction. The Josephson junction is of the SNS, SFS, and S/ $\mathrm{HM} / \mathrm{S}$ type for $V_{\mathrm{ex}} / t=0,1$, and 2.5 , respectively. 
with $\quad \hat{h}_{\boldsymbol{r}, \boldsymbol{r}^{\prime}}=\left[-t \delta_{\left|\boldsymbol{r}-\boldsymbol{r}^{\prime}\right|, 1}+\left(\epsilon_{\boldsymbol{r}}-\mu+4 t\right) \delta_{\boldsymbol{r}, \boldsymbol{r}^{\prime}}\right] \hat{\sigma}_{0}-$ $\mathbf{V}(\boldsymbol{r}) \cdot \hat{\boldsymbol{\sigma}} \delta_{\boldsymbol{r}, \boldsymbol{r}^{\prime}}$ and $\overline{\tilde{c}}_{\boldsymbol{r}}=\left(c_{\boldsymbol{r}, \uparrow}, c_{\boldsymbol{r}, \downarrow}\right)$, where $c_{\boldsymbol{r}, \boldsymbol{\sigma}}^{\dagger}\left(c_{\boldsymbol{r}, \sigma}\right)$ is the creation (annihilation) operator of an electron at $\boldsymbol{r}$ with spin $\sigma=(\uparrow$ or $\downarrow), \overline{\tilde{c}}$ means the transpose of $\tilde{c}, \hat{\sigma}_{l}$ for $l=1-3$ are the Pauli's matrices, and $\hat{\sigma}_{0}$ is $2 \times 2$ unit matrix. The hopping integral $t$ is considered among nearest neighbor sites in both superconductors and ferromagnets. In a ferromagnet, the on-site scattering potentials are given randomly in the range of $-V_{I} / 2 \leq \epsilon_{r} \leq V_{I} / 2$ and the uniform exchange potential is given by $\mathbf{V}(\boldsymbol{r})=V_{\mathrm{ex}} \boldsymbol{e}_{3}$, where $\boldsymbol{e}_{l}$ for $l=1-3$ is unit vector in spin space. The Fermi energy $\mu$ is set to be $2 t$ in a normal metal with $V_{\mathrm{ex}}=0$, while a ferromagnet and a half metal are, respectively, described by $V_{\mathrm{ex}} / t=1$ and 2.5 in Fig. 1(b). Spin-flip scatterings are introduced at $j=1,2, L_{N}-1$, and $L_{N}$, where we choose $\mathbf{V}(\boldsymbol{r})=V_{S} \boldsymbol{e}_{2}$. In superconductors we take $\epsilon_{r}=0$ and $\hat{\Delta}=i \Delta \hat{\sigma}_{2}$, where $\Delta$ is the pair potential in the $s$-wave symmetry.

The Hamiltonian is diagonalized by the Bogoliubov transformation and the Bogoliubov-de Gennes equation is numerically solved by the recursive Green function method $[13,14]$. We calculate the Green function,

$$
\check{G}_{\omega_{n}}\left(\boldsymbol{r}, \boldsymbol{r}^{\prime}\right)=\left(\begin{array}{cc}
\hat{g}_{\omega_{n}}\left(\boldsymbol{r}, \boldsymbol{r}^{\prime}\right) & \hat{f}_{\omega_{n}}\left(\boldsymbol{r}, \boldsymbol{r}^{\prime}\right) \\
-\hat{f}_{\omega_{n}}^{*}\left(\boldsymbol{r}, \boldsymbol{r}^{\prime}\right) & -\hat{g}_{\omega_{n}}^{*}\left(\boldsymbol{r}, \boldsymbol{r}^{\prime}\right)
\end{array}\right),
$$

where $\omega_{n}=(2 n+1) \pi T$ is the Matsubara frequency, $n$ is an integer number, and $T$ is a temperature. The Josephson current is given by $J=-i e t T \sum_{\omega_{n}} \sum_{m=1}^{W} \operatorname{Tr}\left[\check{G}_{\omega_{n}}\left(\boldsymbol{r}^{\prime}, \boldsymbol{r}\right)-\right.$ $\left.\check{G}_{\omega_{n}}\left(\boldsymbol{r}, \boldsymbol{r}^{\prime}\right)\right]$ with $\boldsymbol{r}^{\prime}=\boldsymbol{r}+\boldsymbol{x}$. In this Letter, $2 \times 2$ and $4 \times 4$ matrices are indicated by $\therefore$. and $\therefore$, respectively. The quasiclassical Green function method is a powerful tool to study the proximity effect. However, the quasiclassical Green function cannot be constructed in a half metal because the Fermi energy is no longer much larger than the pair potential for one spin direction. On the other hand, there is no such difficulty in our method. In addition, it is possible to obtain the ensemble average of the Josephson current $\langle J\rangle=\left(1 / N_{S}\right) \sum_{i=1}^{N_{S}} J_{i}$ and its fluctuations $\delta J=$ $\sqrt{\left\langle J^{2}\right\rangle-\langle J\rangle^{2}}$ after calculating the Josephson current for a large number of samples $N_{S}$ with different impurity configurations. These are the advantages of the recursive Green function method. Throughout this Letter we fix the following parameters: $L_{N}=74, W=25, \mu=2 t, V_{I}=$ $2 t$, and $\Delta_{0}=0.005 t$ [15]. This parameter choice corresponds to the diffusive transport regime in the N, F, and HM layers. The results presented below are not sensitive to variations of these parameters.

We first discuss the Josephson current in SFS junctions as shown in Fig. 2(a) for $T=0.1 T_{c}$ where $T_{c}$ is the transition temperature. We assume that the spin-flip scattering at the interfaces is absent (i.e., $V_{S}=0$ ) and fix the phase difference across the junctions $\varphi$ equal to $\frac{\pi}{2}$. The results are normalized by $\left\langle J_{0}\right\rangle$ which is the Josephson current in superconductor/normal metal/superconductor
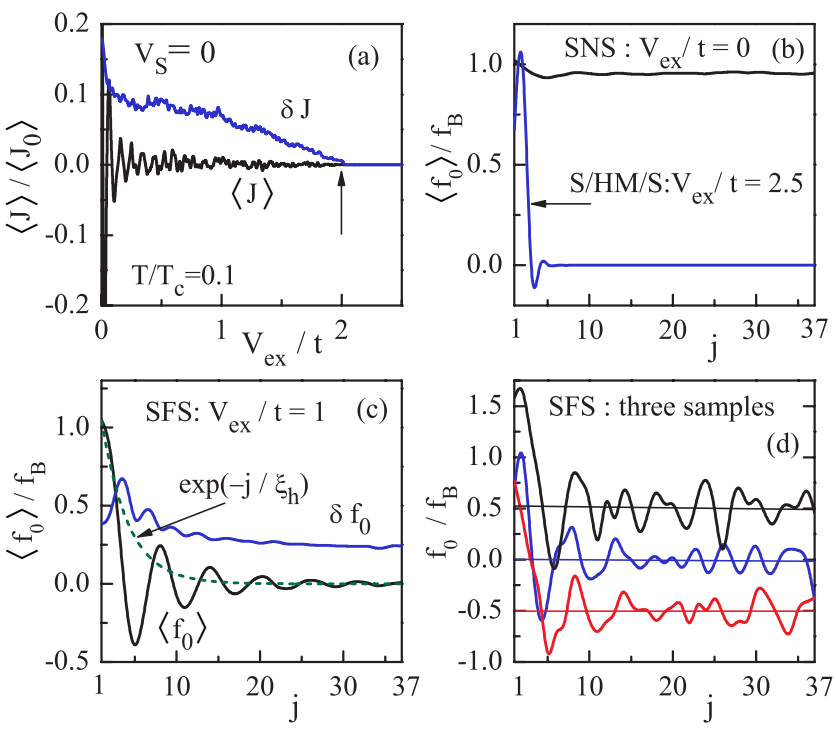

FIG. 2 (color online). (a) The Josephson current versus the exchange potential $V_{\mathrm{ex}}$. At $V_{\mathrm{ex}}=2 t$, a ferromagnet becomes half-metallic as indicated by an arrow. (b) The pairing function of spin-singlet pairs $\left\langle f_{0}\right\rangle$ versus position $j$ for $V_{\mathrm{ex}} / t=0$ (SNS) and 2.5 (S/HM/S); (c) for $V_{\mathrm{ex}} / t=1$ (SFS). (d) The pairing function in three different samples for $V_{\mathrm{ex}} / t=1$, the vertical axis is offset by 0.5 as indicated by the horizontal lines. The spinflip scattering is absent in all panels, $V_{S}=0$.

(SNS) junctions (i.e., $V_{\text {ex }}=0$ ). We define the coherence length $\xi_{h}=\sqrt{D / 2 V_{\mathrm{ex}}} / a_{0}$ measured in units of lattice constant $a_{0}$ with $D$ being the diffusion coefficient. The Josephson current oscillates as a function of $V_{\mathrm{ex}}$ and changes its sign almost periodically. The sign changes of $\langle J\rangle$ correspond to the $0-\pi$ transitions in SFS junctions. At the same time, the amplitude of $\langle J\rangle$ decreases rapidly with increasing $V_{\mathrm{ex}}$. We should pay attention to the relation $\langle J\rangle \ll \delta J$ which means that the Josephson current is not the self-averaging quantity. It is impossible to predict the Josephson current in a single sample $J_{i}$ from the ensemble average $\langle J\rangle$ because $J_{i}$ strongly depends on a microscopic impurity configuration. In fact, the Josephson current flows in a single sample even if $\langle J\rangle=0$ at the transition points. Roughly speaking, $\langle J\rangle$ vanishes because half of samples are 0 junctions and the rest are $\pi$ junctions [16,17]. Since $\langle J\rangle=0, \delta J$ approximately corresponds to the typical amplitude of the Josephson current expected in a single sample. The relation $\langle J\rangle=0$ has different meaning for SFS and $\mathrm{S} / \mathrm{HM} / \mathrm{S}$ cases. In SFS junctions, $\langle J\rangle=0$ at the transition points is the result of the ensemble averaging and the Josephson current remains finite in a single sample. The characteristic temperature and length of a ferromagnet at the $0-\pi$ transitions vary from one sample to another. In $\mathrm{S} /$ $\mathrm{HM} / \mathrm{S}$ junctions at $V_{\mathrm{ex}}=2.5 t$, however, $\langle J\rangle=0$ means vanishing Josephson current even in a single sample [12] because $\langle J\rangle=\delta J=0$.

The origin of large fluctuations of the Josephson current can be understood by considering the behavior of the 
pairing function in a ferromagnet. The pairing function in Eq. (3) can be decomposed into four components,

$$
\frac{1}{W} \sum_{m=1}^{W} \hat{f}_{\omega_{n}}(\boldsymbol{r}, \boldsymbol{r})=i \sum_{\nu=0}^{3} f_{\nu}(j) \hat{\sigma}_{\nu} \hat{\sigma}_{2}
$$

where $f_{0}\left(f_{3}\right)$ is the pairing function of the spin-singlet (spin-triplet) pairs with the spin structure of $(|\uparrow \downarrow\rangle-$ $(+)|\downarrow \uparrow\rangle) / \sqrt{2}$, and the pairing function of $|\uparrow \uparrow\rangle(|\downarrow \downarrow\rangle)$ is given by $f_{\Uparrow}=i f_{2}-f_{1}\left(f_{\Downarrow}=i f_{2}+f_{1}\right)$. In Figs. 2(b) and 2(c), we show $\left\langle f_{0}\right\rangle$ as a function of position $j$ in a diffusive ferromagnet, where $f_{B}$ is the pairing function in bulk superconductor, $\omega_{n}$ is fixed at $0.02 \Delta_{0}, V_{S}=0$, and $\varphi=$ 0 . The junction interface and the center of a ferromagnet correspond to $j=1$ and $j=37$, respectively. In SNS junctions in Fig. 2(b), $\left\langle f_{0}\right\rangle$ is almost constant, which means that spin-singlet Cooper pairs exist everywhere in a normal metal. We confirmed the relation $\left\langle f_{0}\right\rangle>\delta f_{0}$ in SNS junctions. On the other hand, in SFS junctions as shown in Fig. 2(c), the average $\left\langle f_{0}\right\rangle$ decreases exponentially with $j$ according to $\exp \left(-j / \xi_{h}\right)$ as indicated by a broken line. The fact that $\delta f_{0}$ remains finite at the center of a ferromagnet means that the spin-singlet pairs penetrate far beyond $\xi_{h}$ even though $\left\langle f_{0}\right\rangle \sim 0$ there. In Fig. 2(d), we show the pairing function in SFS junctions for three samples with different impurity distribution. The pairing functions are in phase near the interface $\left(j \leq \xi_{h}\right)$, whereas they are out of phase far from the interface. We obtain the relation $\delta f_{0} \propto$ $e^{-j / \xi_{T}}$ with $\xi_{T}=\sqrt{D / 2 \omega_{n}}$ in agreement with Ref. [16]. Thus we conclude that spin-singlet Cooper pairs do exist in a single sample of ferromagnet even for $j \gg \xi_{h}$ and the mesoscopic fluctuations of the pairing function provide the origin of the large fluctuations in the Josephson current. In $\mathrm{S} / \mathrm{HM} / \mathrm{S}$ junctions for $V_{\mathrm{ex}}=2.5 t$ as shown in Fig. 2(b), $\left\langle f_{0}\right\rangle$ vanish for $j \gg 1$. We also confirmed $\delta f_{0}=0$ for $j \gg 1$ at the same time. These results indicate the absence of spin-singlet Cooper pairs in a half metal.

The relation $\langle J\rangle \ll \delta J$ is the characteristic feature of the Josephson current in diffusive SFS junctions with $V_{\mathrm{ex}} \gg$ $\Delta_{0}$. This feature, however, is drastically changed by the spin-flip scattering at the interfaces. In Figs. 3(a) and 3(b), we show $\langle J\rangle$ and $\delta J$ as a function of the spin-flip potential $V_{S}$ for $V_{\mathrm{ex}} / t=1$ and 2.5 , respectively. In both cases (a) and (b), we find that $|\langle J\rangle| \geq \delta J$ for $V_{S} \geq 0.3 t$. The Josephson current becomes self-averaging in the presence of the spinflip scattering. The reason can be explained by the pairing functions of equal-spin pairs shown in Figs. 3(c) and 3(d), where $f_{\nu}$ is plotted as a function of position $j$. Here we show $\delta f_{0}$ instead of $\left\langle f_{0}\right\rangle$ because $\delta f_{0} \gg\left\langle f_{0}\right\rangle$. The characteristic behavior of $\left\langle f_{3}\right\rangle$ and $\delta f_{3}$ are the same with those of $\left\langle f_{0}\right\rangle$ and $\delta f_{0}$, respectively. In both cases (c) and (d), $\left\langle f_{\Uparrow}\right\rangle$ becomes much larger than $\delta f_{0}$ because the pairing function $f_{\Uparrow}$ does not change sign for various impurity configurations. Thus the Josephson current becomes selfaveraging as shown in Figs. 3(a) and 3(b).
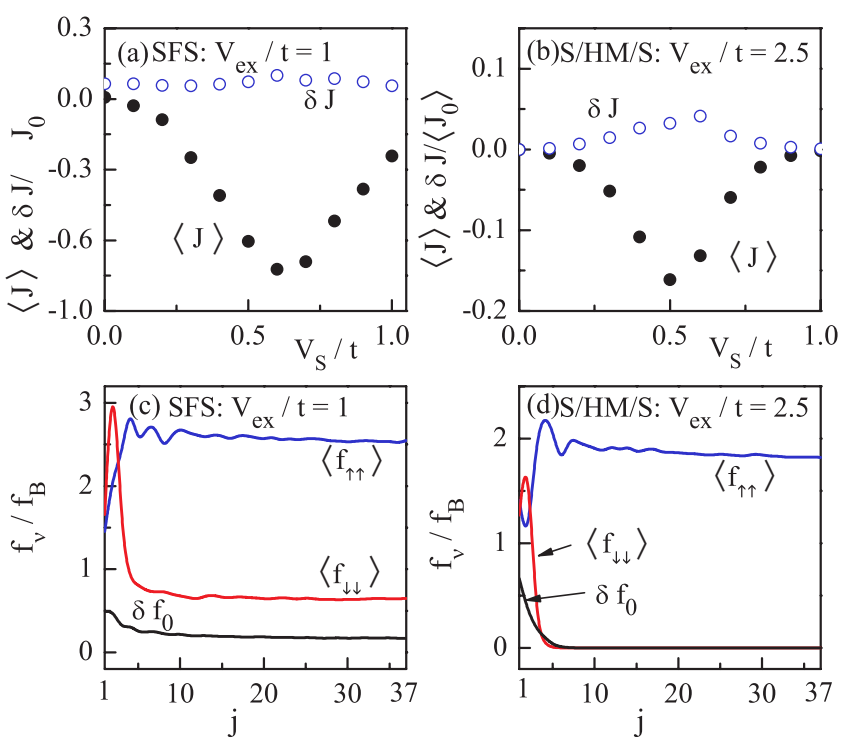

FIG. 3 (color online). (a) The Josephson current and its fluctuations at $T=0.1 T_{c}$ and $\varphi=\frac{\pi}{2}$ as a function of the interface spin-flip scattering $V_{S}$ for $V_{\mathrm{ex}} / t=1$ and (b) for $V_{\mathrm{ex}} / t=2.5$. The pairing functions versus position $j$ in a ferromagnet (c) and in a half metal (d) at $V_{s}=0.4 t, \varphi=0$, and $\omega_{n}=0.02 \Delta_{0} .\left\langle f_{\uparrow}\right\rangle$ and $\left\langle f_{\Downarrow \downarrow}\right\rangle$ are the pairing functions of equal spin pairs, and $\delta f_{0} \gg$ $\left\langle f_{0}\right\rangle$ indicates the amplitude of pairing function for spin-singlet pairs.

Finally, we address an unusual symmetry property of Cooper pairs in S/HM/S junctions. In Fig. 4(a), we show $\left\langle f_{\Uparrow \uparrow}\right\rangle$ as a function of $\omega_{n}$, where $j=37, V_{S}=0.2 t, \varphi=0$, and $V_{\mathrm{ex}}=2.5 t$. For comparison, we also show $\left\langle f_{0}\right\rangle$ on the normal side of a SNS junction. The pairing function $\left\langle f_{0}\right\rangle$ in a normal metal is the even function of $\omega_{n}$, whereas $\left\langle f_{\Uparrow}\right\rangle$ in a half metal is the odd function of $\omega_{n}$ [6]. The pairing function obeys the Pauli's rule

$$
\hat{f}_{\omega_{n}}\left(\boldsymbol{r}, \boldsymbol{r}^{\prime}\right)=-\overline{\hat{f}}_{-\omega_{n}}\left(\boldsymbol{r}, \boldsymbol{r}^{\prime}\right),
$$

where $\overline{\hat{f}}$ denotes the transpose of $\hat{f}$ meaning the interchange of spins. It is well known that ordinary evenfrequency pairs are classified into two symmetry classes:
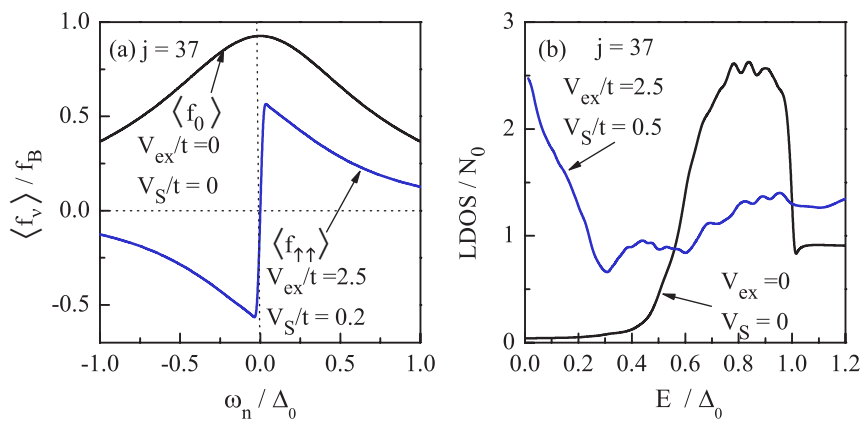

FIG. 4 (color online). (a) Dependences of the pairing functions on $\omega_{n}$. (b) The local density of states at $j=37$ in a half metal at $V_{S}=0.5 t$ and in a normal metal at $V_{S}=0$. 
spin-singlet even-parity and spin-triplet odd-parity. In the former case, the negative sign arises due to the interchange of spins, while in the latter case due to $\boldsymbol{r} \leftrightarrow \boldsymbol{r}^{\prime}$. In the present calculation, all components on the right hand side of Eq. (4) have the $s$-wave symmetry. The pairing functions are isotropic in both real and momentum spaces due to diffusive impurity scattering [18]. As a result, $f_{\Uparrow \uparrow}$ must be the odd function of $\omega_{n}$ to obey the Pauli's rule. Both evenand odd-frequency pairs are mixed in ferromagnets as shown in Fig. 3(c). The fraction of odd-frequency pairs depends on parameters such as the exchange potential and the spin-flip scattering. On the other hand, in a diffusive half metal all Cooper pairs have the odd-frequency character, which causes drastic change in the quasiparticle density of states.

The density of states is given by $N(E, j)=-\frac{1}{\pi} \frac{1}{W} \times$ $\sum_{m=1}^{W} \operatorname{Im} \operatorname{Tr} \breve{G}_{E+i \gamma}(\boldsymbol{r}, \boldsymbol{r})$, where $\gamma$ is a small imaginary part chosen to be $0.05 \Delta_{0}$ in the following. In Fig. 4(b), the local density of states (LDOS) at $j=37$ is shown, where $\varphi=0$ and $N_{0}$ is the density of states in the normal state at $V_{\mathrm{ex}}=0$. For comparison, we show LDOS on the normal side of the SNS junction with $V_{S}=0$ which has a minigap at $E<E_{\mathrm{Th}} \sim 0.3 \Delta_{0}$, where $E_{\mathrm{Th}}$ is the Thouless energy. In contrast to that, LDOS in a half metal has a peak at the Fermi energy, its width is characterized by $E_{\mathrm{Th}}$. This peak is generated at the spin active interface [19] and is transferred into a half metal due to the long range property of odd-frequency pairing function. The peak is much stronger than the enhancement of the LDOS found in weak ferromagnets $[3,10,20,21]$. The peak shape is almost independent of position in a half metal. In the SF junctions [20], on the other hand, the LDOS has an oscillatory peak/dip structure at $E=0$, which rapidly decays with the distance from the SF interface. Therefore the large peak at $E=0$ in LDOS is robust and direct evidence of the odd-frequency pairing in half metals. Scanning tunneling spectroscopy could be used to detect such a peculiar pairing state.

In conclusion, we have studied the Josephson effect in superconductor/diffusive ferromagnet/superconductor junctions by using the recursive Green function method. The Josephson current in these junctions basically is not self-averaging because the spin-singlet Cooper pairs penetrating into ferromagnets far beyond $\xi_{h}$ cause large fluctuations of the pairing function. In the presence of spin-flip scattering at the interfaces, the equal-spin odd-frequency pairs drastically suppress the fluctuations. When ferromagnets are half-metallic, all Cooper pairs have the oddfrequency property. As a result, the low energy peak in the quasiparticle density of states in a half metal exists and could be probed by scanning tunneling spectroscopy.
We acknowledge helpful discussions with J. Aarts, T. M. Klapwijk, Yu. Nazarov and A.F. Volkov. This work was partially supported by the Dutch FOM, the NanoNed program under Grant No. TCS7029 and Grant-in-Aid for Scientific Research from The Ministry of Education, Culture, Sports, Science and Technology of Japan (Grants No. 18043001, No. 17071007, and No. 17340106).

Note added. - After submission, we learned about two related Letters where similar predictions were made [22].

[1] P. Fulde and R. A. Ferrell, Phys. Rev. 135, A550 (1964).

[2] A. I. Larkin and Y. N. Ovchinnikov, Sov. Phys. JETP 20, 762 (1965).

[3] A. I. Buzdin, L. N. Bulaevskii, and S. V. Panyukov, JETP Lett. 35, 179 (1982).

[4] A. I. Buzdin, Rev. Mod. Phys. 77, 935 (2005).

[5] V.T. Petrashov, V.N. Antonov, S. Maksimov, and R. Shaikhaidarov, JETP Lett. 59, 551 (1994).

[6] F. S. Bergeret, A. F. Volkov, and K. B. Efetov, Phys. Rev. Lett. 86, 4096 (2001); Rev. Mod. Phys. 77, 1321 (2005).

[7] A. Kadigrobov, R. I. Shekhter, and M. Jonson, Europhys. Lett. 54, 394 (2001).

[8] V. V. Ryazanov, V. A. Oboznov, A. Yu. Rusanov, A. V. Veretennikov, A. A. Golubov, and J. Aarts, Phys. Rev. Lett. 86, 2427 (2001).

[9] T. Kontos, M. Aprili, J. Lesueur, F. Genet, B. Stephanidis, and R. Boursier, Phys. Rev. Lett. 89, 137007 (2002).

[10] A. A. Golubov, M. Yu. Kupriyanov, and E. I'ichev, Rev. Mod. Phys. 76, 411 (2004).

[11] R.S. Keizer, S. T.B. Goennenwein, T. M. Klapwijk, G. Miao, G. Xiao, and A. Gupta, Nature (London) 439, 825 (2006).

[12] M. Eschrig, J. Kopu, J. C. Cuevas, and G. Schon, Phys. Rev. Lett. 90, 137003 (2003).

[13] A. Furusaki, Physica (Amsterdam) 203B, 214 (1994).

[14] Y. Asano, Phys. Rev. B 63, 052512 (2001).

[15] The mean free path $\ell$ and the coherence length $\xi_{0}$ are about 6 and 100 lattice constant, respectively. Qualitatively the same results as Figs. 2-4 are confirmed also for $\xi_{0} \sim 10$.

[16] A. Yu. Zyuzin, B. Spivak, and M. Hruska, Europhys. Lett. 62, 97 (2003).

[17] Y. Asano, Phys. Rev. B 64, 014511 (2001); J. Phys. Soc. Jpn. 71, 905 (2002).

[18] Y. Tanaka and A. A. Golubov, Phys. Rev. Lett. 98, 037003 (2007).

[19] M. Fogelstrom, Phys. Rev. B 62, 11812 (2000).

[20] T. Kontos, M. Aprili, J. Lesueur, and X. Grison, Phys. Rev. Lett. 86, 304 (2001).

[21] T. Yokoyama, Y. Tanaka, and A. A. Golubov, Phys. Rev. B 72, 052512 (2005).

[22] V. Braude and Yu. V. Nazarov, Phys. Rev. Lett. 98, 077003 (2007); M. Eschrig and T. Lofwander, cond-mat/0612533. 